\title{
Agarose-Based System for Separation of Short Tandem Repeat Loci
}

\author{
Hugh W. White and Noriko Kusukawa \\ FMC BioProducts, Rockland, ME, USA
}

BioTechniques 22:976-980 (May 1997)

\begin{abstract}
Short tandem repeats (STRS) are traditionally analyzed on large polyacrylamide electrophoresis gels. We demonstrate in this study that a small (10-cm-long, 1-mm-thick) agarose gel is sufficient for analysis of multiplexed samples for several commonly used STR loci. A system was developed using a high-resolution agarose, MetaPhor ${ }^{\circledR}$. Within an hour of electrophoresis, sufficient resolution was obtained to allow discrimination of triple-multiplexed STR loci. We show that this agarose is capable of resolving di-as well as tetranucleotide ladders. Using PCR conditions similar to those routinely used with sensitive detection systems, we found that direct staining of gels with $S Y B R^{\circledR}$ Green I stain was comparable with silver staining, autoradiography or fluorescently tagged primers for sample detection and was considerably easier. This procedure significantly simplifies STR analysis and is much faster than many standard protocols.
\end{abstract}

\section{INTRODUCTION}

Short tandem repeats (STRs) are markers widely used for genetic linkage mapping, medical testing and forensic applications $(1,5,7)$. The majority of these markers are analyzed as DNA fragments in the size range of 100-350 bp with repeating units of 2, 3, 4 or 5 bases. The relative ease, or difficulty, of electrophoretic discrimination between different alleles is determined by the size of the repeating units, the distribution of the number of repeats within the population, and the specificity and efficiency of polymerase chain reaction (PCR), which dictate the quality of the resulting analytes. STRs are usually analyzed on a large denaturing DNA sequencing gel, as it provides the best resolution attainable in electrophoresis. However, these gels are cumbersome to prepare, unstable and often variable, so that standardization of data within or between laboratories may be difficult. Methods for detection are also limited because the DNA is denatured into single-strand- ed form, which necessitates the use of radioisotopes, silver staining or laser-based fluorescence detection instruments. A simpler, faster and more reproducible method for electrophoresis and visualization would be preferable if sufficient resolution and sensitivity could be attained. In this study, we used non-denaturing agarose gels ( $1 \mathrm{~mm}$ thick) that were cast in plastic minicassettes for analysis of tetranucleotide STRs. A high-resolution agarose, MetaPhor ${ }^{\circledR}$ (FMC BioProducts, Rockland, ME, USA), was used, and good resolution was achieved within an hour of electrophoresis. STR loci were amplified by PCR cycle numbers commonly used for samples intended for high-sensitivity detection methods such as silver staining or radioisotopic detection. The amount of DNA prepared by this procedure was usually below the detection limit of ethidium bromide $(\mathrm{EtdBr})$; therefore, a more sensitive stain, SYBR ${ }^{\circledR}$ Green I stain (FMC BioProducts), was used for visualization of the gel. To allow clear analysis of multiple loci in one lane, it was important to perform PCR at lower cycle numbers to minimize nonspecific bands and subsequent interpretation problems. Three STR loci, all consisting of tetranucleotide repeats, were analyzed in one lane. The results confirmed a familial linkage identity of the samples used. In addition, we showed that the same agarose is capable of 2-bp discrimination and may be used, with SYBR Green I staining, to analyze dinucleotide STRs within the limits imposed by the amplification process. With this system, most of the commonly used STR loci can be accurately analyzed more easily and with higher throughput than by current techniques.

\section{MATERIALS AND METHODS}

\section{PCR Amplification}

Amplifications of tetranucleotide repeat STR loci HPRTB, FESFPS and vWF were performed in a PTC-100 ${ }^{\mathrm{TM}}$ Mini- 
Cycler (MJ Research, Watertown, MA, USA) using GenePrint $^{\mathrm{TM}}$ STR systems (Promega, Madison, WI, USA) under amplification conditions recommended by the manufacturer and AmpliTaq ${ }^{\circledR}$ DNA Polymerase (Perkin-Elmer, Norwalk, CT, USA). Amplifications of the dinucleotide repeat locus D6S264 were performed using commercially available primers (Research Genetics, Huntsville, AL, USA). Genomic DNA samples (25-150 ng/25- $\mu \mathrm{L}$ reaction) from CEPH families 884 or 1333 (BIOS Laboratories, New Haven, CT, USA) were used as templates for the amplifications.

In experiments where EtdBr was used for detection of tetranucleotide repeats, amplifications were performed for 35 PCR cycles to reliably produce sufficient DNA for detection. Thirty cycles were used for detection with SYBR Green I. Dinucleotide repeats were obtained by 35 PCR cycles using the amplification profile of denaturation at $94^{\circ} \mathrm{C}$, annealing at $55^{\circ} \mathrm{C}$ and extension at $72^{\circ} \mathrm{C}$. Following amplification, 0.25 vol of concentrated loading dye (30\% Ficoll ${ }^{\circledR} 400$ [Pharmacia Biotech, Piscataway, NJ, USA], 100 mM EDTA, 0.1\% bromphenol blue) was added to the reactions, and aliquots $(3-10 \mu \mathrm{L})$ of the mixture were used for electrophoretic analysis.

\section{Gel Preparation}

MetaPhor agarose solutions were prepared at $4 \%$ in $1 \times$ TBE ( $89 \mathrm{mM}$ Tris-borate, $2.0 \mathrm{mM}$ EDTA, $\mathrm{pH}$ 8.3). Vertical gels were cast using a 60-cc disposable syringe with a 16gauge needle to dispense the "hot" $\left(70^{\circ}-80^{\circ} \mathrm{C}\right)$ agarose solution directly into ready-to-use plastic minicassettes (Novex, San Diego, CA, USA) or preassembled glass cassettes prewarmed to $65^{\circ} \mathrm{C}$ to prevent premature gelation. Minigels were placed at $4{ }^{\circ} \mathrm{C}$ immediately following casting and were ready to use $20 \mathrm{~min}$ after casting. Larger gels $(18 \times 24 \mathrm{~cm})$ were allowed to gel at room temperature for $20-30 \mathrm{~min}$ and then placed at $4^{\circ} \mathrm{C}$ for $20 \mathrm{~min}$ to ensure complete gelation.

\section{Gel Electrophoresis}

Vertical agarose gel electrophoresis was performed using a minichamber (Novex XCELL II) or the Hoefer SE 660 Chamber for the larger $(18 \times 24 \mathrm{~cm})$ gels (Hoefer Pharmacia Biotech, San Francisco, CA, USA). Electrophoresis of minigels in the Novex unit was conducted for $1.0 \mathrm{~h}$ at constant voltage $(30-35 \mathrm{~V} / \mathrm{cm})$ using $1 \times$ TBE running buffer. The buffer was prechilled to $4^{\circ}-6^{\circ} \mathrm{C}$ to maintain uniformity of the band pattern across the gel. The buffer temperature typically rose to $18^{\circ}-20^{\circ} \mathrm{C}$ during electrophoresis. Electrophoresis of $24-\mathrm{cm}-$ long gels in the Hoefer unit was conducted for $4 \mathrm{~h}$ at constant voltage $(21 \mathrm{~V} / \mathrm{cm})$ using $1 \times$ TBE running buffer. The nominal voltage gradients were determined by dividing the applied voltage by the length of the gel cassettes in the apparatus (17).

Following electrophoresis of the minigels, the cassettes were disassembled, and the gels were carefully transferred to a small container by sliding them from the plastic plate. Gels were stained for $10 \mathrm{~min}$ with either $1 \mu \mathrm{g} / \mathrm{mL}$ EtdBr prepared in distilled water or a 1:10000 dilution of SYBR Green I stock solution prepared in $1 \times$ TBE. Staining of large gels with SYBR Green I was performed by disassembling the cassette, leaving the gel on one glass plate. A small volume of SYBR Green I stain was then carefully pipetted onto the gel surface, and the staining was allowed to proceed for $10-15 \mathrm{~min}$. Gels were rinsed with distilled water, visualized on a transilluminator (Fotodyne, Hartland, WI, USA) using 304-nm UV illumination, and photographed using a Polaroid MP-4 camera (Cambridge, MA, USA), Type 55 Positive-Negative film and appropriate filters. Gels were also scanned on the FluorImager $^{\text {TM }}$ SI (Molecular Dynamics, Sunnyvale, CA, USA).

\section{Analysis of Allelic Sizes}

Allelic sizes and resolution were estimated by comparison to DNA size markers: pBR322 MspI digest (New England Biolabs, Beverly, MA, USA), 20-bp ladder (Gensura, Del Mar, CA, USA) and di- or tetranucleotide ladders prepared by ligation of mixtures of phosphorylated linkers.

\section{RESULTS}

Figure 1 shows examples of STR separations on MetaPhor agarose minigels using a tetranucleotide repeat locus, FESFPS, as a model system. Allelic bands that differ by 4 (lanes 1 and 2), 8 (lane 3) or 12 (lane 4) bp are clearly resolved by this gel system. Samples amplified by a 35 -cycle PCR yielded sufficient material for detection by EtdBr staining, but also showed nonallelic bands and non-uniform amplification of the larger and smaller alleles present in each sample (Figure 1A). Reducing the cycle number to 30 effectively eliminated most of the artifacts and generated primarily the allelic bands, which were analyzed easily by staining with SYBR Green I (Figure 1B).

Figure 2 demonstrates use of the agarose minigel system for separation of multiplex STR samples containing products amplified from three loci (HPRTB, FESFPS and vWF) ranging in size from 140 to $300 \mathrm{bp}$. These products were amplified using genomic DNA samples from three generations of a well-characterized CEPH family. With one exception (only one allelic band for the HPRTB locus was detected in lane 5), patterns for all three loci are consistent with the familial assignment. These data confirm that the agarose gel system is indeed capable of tracking allelic inheritance. The results also show that formation of heteroduplex products varies among

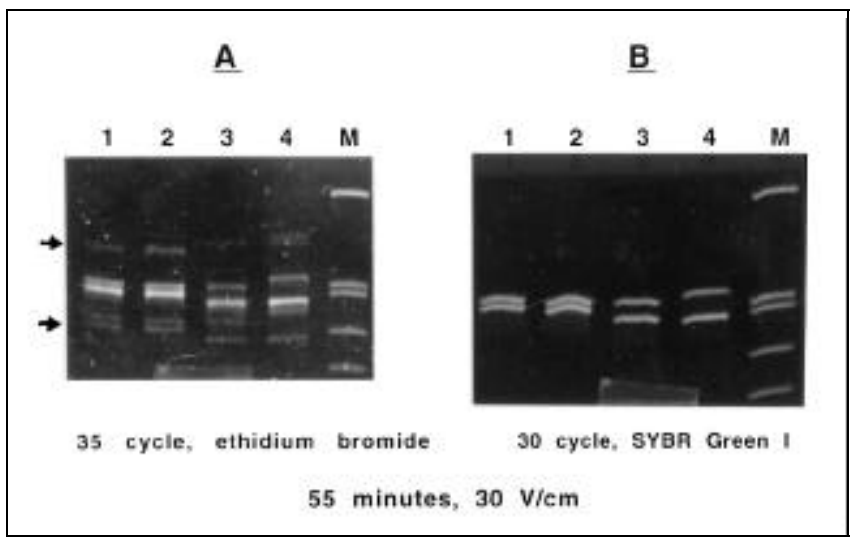

Figure 1. Separation of amplification products from the FESFPS locus on agarose gels. Amplification products from unrelated individuals were separated on $4 \%$ MetaPhor agarose gels. PCRs, electrophoresis conditions and post-electrophoretic staining were as indicated. Both gels used pBR322 MspI digest (New England Biolabs) as a size standard (M). The bands shown in the marker lanes (from left to right) are: 307, 242, 238, 217 and $201 \mathrm{bp}$. Arrows indicate artifactual bands seen above and below allelic bands in Panel A. 
STR loci. Slowly migrating heteroduplex bands were seen for some samples at the HPRTB and vWF loci but were not detected for the FESFPS locus.

In Figure 2B, one fifth of each sample used in Figure 2A was loaded on the gel, stained with SYBR Green I after electrophoresis and scanned with the FluorImager SI. The results demonstrate the increased sensitivity obtained with 488-nm excitation of SYBR Green I-stained samples.

To further demonstrate the limits of resolution of this agarose gel system, we prepared 2- and 4-bp ladders, amplified samples from the dinucleotide locus D6S264 and analyzed these DNAs on a 24-cm gel with the FluorImager SI Scanning System. The results are shown in Figure 3. Clear resolution of 2-bp intervals is seen, up to about $200 \mathrm{bp}$ in the ladders. Allelic bands (the most intensely stained bands) were distinctly resolved, as were the more faintly staining stutter bands seen above and below the allelic bands in the amplified samples. Comparison of the spacing between rungs of the 2bp ladder and that of allelic bands and stutter bands demonstrates that the gel system is clearly capable of resolving 2-bp; i.e., single allele size differences and shows that the samples in lanes 4 and 5 are examples of homozygotes differing in size by a single repeat. Since stutter bands are the source of the most intractable problems of interpretation, the limit to analysis of such loci probably lies more in the design of the PCR system than in the resolving power of the gel matrix.

\section{DISCUSSION}

Several reports have described the use of non-denaturing polyacrylamide gels $(4,11,12,14)$ or horizontal agarose gels (16) to circumvent the use of large sequencing gels for STR analyses. The omission of denaturants from the gel allows the DNA to be analyzed in double-stranded form. Double-stranded DNA migrates faster than single-stranded DNA in electrophoresis and is also more easily detected. However, in the non-denaturing polyacrylamide gels, double-stranded DNA samples that contain secondary structures or small sequence differences may migrate anomalously $(3,15)$. While this may be tolerable in simple STR analyses, the interpretation of multiplexed samples may be complicated by overlaps between bands of different loci. In agarose, the difference in migration of hetero- and homoduplex fragments is less extreme. As seen in Figure 2, heteroduplex bands are visible but do not overlap with other loci. In this vertical minigel format, the three STR loci were clearly resolved in one lane despite the short distance available for separation.

The effect of fewer PCR cycles on the quality of the resulting DNA was dramatic (Figure 1). A 35-cycle PCR produced samples with many artifacts and also amplified unequal amounts of the two alleles, which would be expected at 1:1 ratio in the heterozygote samples. Shortening the amplification by 5 cycles corrected both problems, as reported previously $(10,13)$. The specificity of amplification and the extent of heteroduplex formation clearly vary with different STR loci, the primers chosen and on the quality and quantity of the template. A further reduction in the number of PCR cycles may be useful for some loci.

This reduced-cycles approach requires that the detection method be highly sensitive. SYBR Green I stain has a detection limit of $50 \mathrm{pg}$ per band using a 304-nm transilluminator, approximately five times more sensitive than ethidium bromide under similar conditions. Also, the sensitivity of the detection can be increased by the use of a 254-nm UV light source and further by the use of gel-imaging systems that ex-

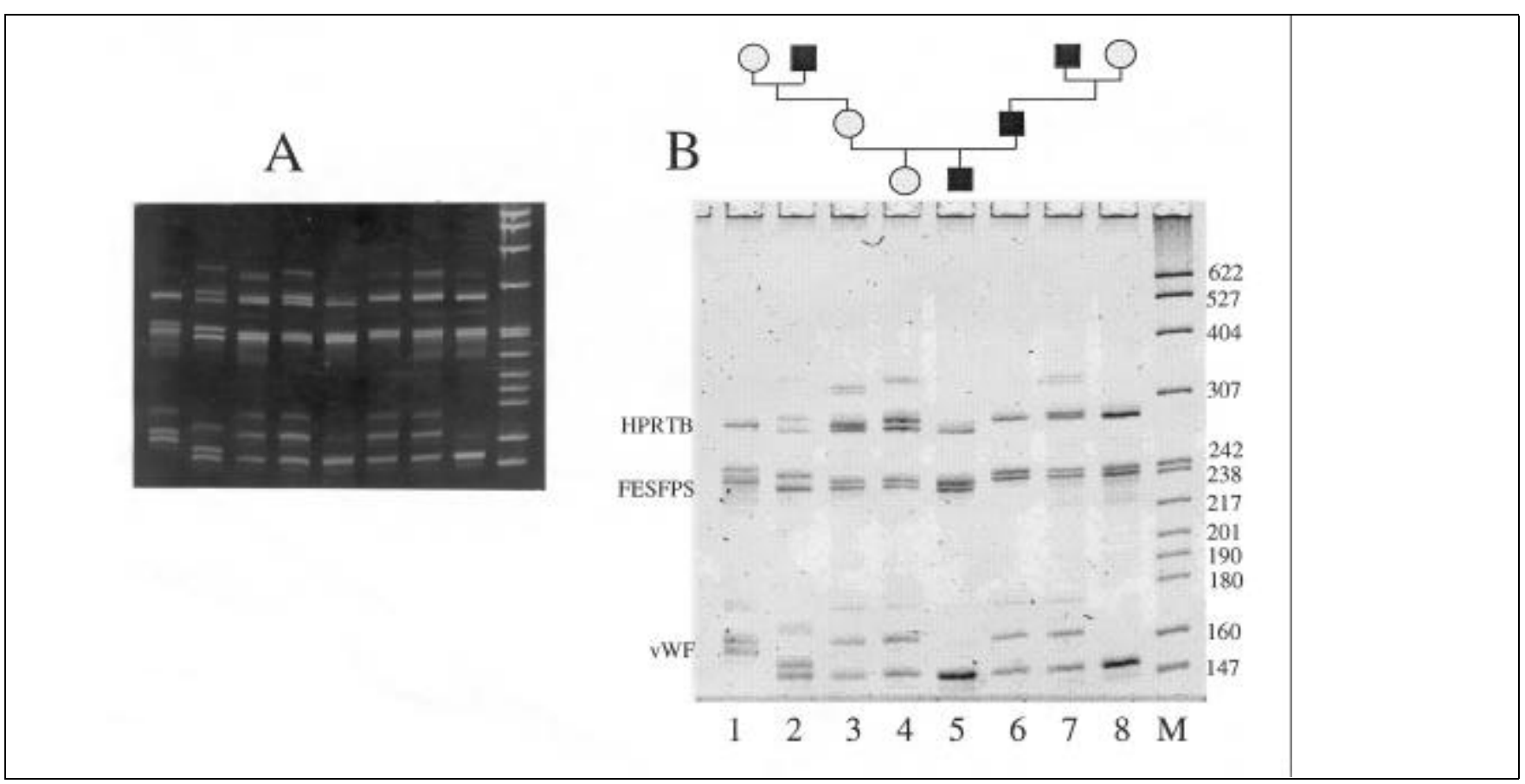

Figure 2. Separation of multiplexed STRs using 4\% MetaPhor agarose minigels. Samples from eight individuals from CEPH family 1333 were amplified at the HPRTB, FESFPS and vWF loci. Details of the sample identity are included in the pedigree shown above Panel B. Individual amplification reactions were combined and resolved simultaneously by electrophoresis on MetaPhor agarose minigels run at $300 \mathrm{~V}$ for 55 min (Panel A) or 70 min (Panel B). Samples were loaded on to the two gels in the same order, but the amount loaded on gel A was five times higher than that used on gel B. SYBR Green I was used for staining both gels. Detection in Panel A was performed using UV transillumination, and detection in Panel B was by $488 \mathrm{~nm}$ excitation using the FluorImager SI. Lanes labeled M contained size markers (pBR322 MspI digest). The sizes of bands shown in the marker lane are shown in Panel B. 
cite the stain with a 488-nm, argon ion-based laser. The FluorImager SI detected as little as $15 \mathrm{pg}$ of DNA separated on a 1$\mathrm{mm}$-thick 4\% MetaPhor agarose gel (data not shown), confirming that SYBR Green I can replace the more complex and time-consuming silver staining or autoradiography. Excitation at $488 \mathrm{~nm}$ allows further reduction in PCR cycles to optimize the quality of STR analytes.

Dinucleotide repeat units are the most commonly found motifs in the human genome. However, despite their potential usefulness for mapping, their analysis is often confused by extra "stutter bands" generated during amplification $(6,9)$. Various methods have been devised to minimize detection of these artifacts, requiring extra steps such as end-labeling or even blotting and hybridization (9). For these reasons, multiple loci have been analyzed using STR loci with longer repeat units. With the agarose minigel, we successfully resolved multiplex combinations of commonly used STR loci including HPRTB, FESFPS and vWF (Figure 2), and CSF1PO, TPOX and THO1 (data not shown), all of which contain four nucleotide repeat units.

The theoretical resolution capacity of the minigels used in this study averaged $3.3 \mathrm{bp}$ within the size range of 160-307 bp according to the calculation method of Lerman and Sinha (8), with more resolution of smaller STR fragments and less resolution of larger fragments. When the length of the gel was extended to $24 \mathrm{~cm}$ (Figure 3), the resolution capacity of the gel over the same size interval averaged $1.9 \mathrm{bp}$, confirming that the longer gel is capable of resolving 2-bp intervals. The added advantage of the sensitive SYBR Green I stain allows cleaner amplification with fewer cycles, thereby regaining the usefulness of dinucleotide STRs for mapping.

The emergence of high-resolution and high-sensitivity imaging systems may allow further miniaturization of gels to become a viable strategy for high-throughput analyses. The preparation of agarose gels in vertical plastic cassettes is a surprisingly robust procedure and faster than the preparation of polyacrylamide gels of the same size. Furthermore, the chemical stability of agarose allows one to prepare large numbers of gels in advance, which is useful for any application requiring consistent gel-to-gel performance or standardization.



Figure 3. Resolution of dinucleotide repeats on MetaPhor agarose. Samples from unrelated individuals were amplified at the D6S264 locus. Amplification products were separated by electrophoresis on a $24-\mathrm{cm}-$ long $4 \%$ MetaPhor agarose gel run at $500 \mathrm{~V}$ for $4 \mathrm{~h}$. DNA was detected by post-electrophoretic staining with SYBR Green I stain and scanning on the FluorImager SI. Lanes were loaded as follows: lane 1, 4-bp ladder; lane 2, 20-bp ladder; lane 3, 2-bp ladder; lanes 4 and 5, amplification products. 


\section{ACKNOWLEDGMENTS}

We would like to thank Valerie Burland, Mark Garner and Douglas Robinson for their suggestions and critical readings of this manuscript. We also thank Valerie Burland for suggestions and help with revision of the manuscript.

\section{REFERENCES}

1.Alford, R.L. and C.T. Caskey. 1994. DNA analysis in forensics, disease and animal/plant identification. Curr. Opin. Biotech. 5:29-33.

2.Edwards, A., A. Civetello, H.A. Hammond and C.T. Caskey. 1991. DNA typing and genetic mapping with trimeric and tetrameric tandem repeats. Am. J. Hum. Genet. 49:746-756.

3.Eng, B., P. Ainsworth and J.S. Waye. 1994. Anomalous migration of PCR products using nondenaturing polyacrylamide gel electrophoresis: the amelogenin sex-typing system. J. Forensic Sci. 39:1356-1358.

4.Gen, M.W., P.J. Parry, Y. Ning and G.A. Evans. 1993. Highly polymorphic tetramer repeat (GATA)n on human chromosome 11p15.3. Genomics 17:770-772.

5.Hammond, H.A. and C.T. Caskey. 1994. Human DNA fingerprinting using short tandem repeat loci. Methods Mol. Cell. Biol. 5:78-86.

6.Hauge, X.Y. and M. Litt. 1993. A study of the origin of 'shadow bands' seen when typing dinucleotide repeat polymorphisms by the PCR. Hum. Mol. Genet. 2:411-415.

7.Hearne, C.M., S. Ghosh and J.A. Todd. 1992. Microsatellites for linkage analysis of genetic traits. Trends Genet. 8:288-294.

8.Lerman, L.S. and D. Sinha. 1990. Describing resolution in gel electrophoresis, p. 1-8. In E. Lai and B.W. Birren (Eds.), Electrophoresis of Large DNA Molecules. Cold Spring Harbor Laboratory Press, Cold Spring Harbor, NY.

9.Litt, M., X. Hauge and V. Sharma. 1993. Shadow bands seen when typing polymorphic dinucleotide repeats: some causes and cures. BioTechniques 15:280-284

10.McCulloch, R.K., C.S. Choong and D.M. Hurley. 1995. An evaluation of competitor type and size for use in the determination of mRNA by competitive PCR. PCR Methods Appl. 4:219-226.

11.Mellersh, C. and J. Sampson. 1993. Simplifying detection microsatellite length polymorphisms. BioTechniques 15:582.

12.Morin, P.A. and D.G. Smith. 1995. Nonradioactive detection of hypervariable simple sequence repeats in short polyacrylamide gels. BioTechniques 19:223-228.

13.Schneeberger, C., P. Speiser, F. Kury and R. Zeillinger. 1995. Quantitative detection of reverse transcriptase-PCR products by means of a novel and sensitive DNA stain. PCR Methods Appl. 4:234-238.

14.Scrimshaw, B.J. 1992. A simple nonradioactive procedure for visualization of $(\mathrm{dC}-\mathrm{dA}) n$ dinucleotide repeat length polymorphisms. BioTechniques 13:189.

15.Stellwagen, A. and N.C. Stellwagen. 1990. Anomalously slow electrophoretic mobilities of DNA restriction fragments in polyacrylamide gels are not eliminated by increasing the gel pore size. Biopolymers 30:309324.

16.Stern, C.M., K.M. Lobato and M.S. Schanfield. 1994. Development of simple, inexpensive multiplex small repeat (STR)-AFLP typing systems. Proceedings from the Fifth International Symposium on Human Identification: 25 .

17.Wagner, L. and E. Lai. 1994. Separation of large DNA molecules with high voltage pulsed field gel electrophoresis. Electrophoresis 15:10781083 .

Address correspondence to Hugh W. White, FMC BioProducts, 191 Thomaston Street, Rockland, ME 04841, USA. Internet:hugh_white@fmc.com 